\title{
APPROXIMATE SOLUTIONS TO SOME STATIC AND DYNAMIC OPTIMAL STRUCTURAL DESIGN PROBLEMS*
}

\author{
BY RAYMOND H. PLAUT (Brown University)
}

Problems of optimal structural design for minimum deflection, maximum fundamental frequency and maximum stability are considered in this note. The optimality conditions for these problems consist of nonlinear differential equations which usually require a numerical solution [1-16]. An alternate approach based on the Ritz method is presented here, in which one works with an approximate expression for the objective function rather than the optimality equation. A few examples are considered and some new results are presented.

For simplicity the analysis will be restricted to elastic beam-columns subjected to distributed transverse loads and axial loads. Let $x$ be the axial coordinate $(0 \leq x \leq l)$, $m(x)$ the mass per unit length and $s(x)$ the bending stiffness. A sandwich cross-section is assumed, with a linear mass-stiffness relation

$$
m(x)=a^{2}+b^{2} s(x) .
$$

Beams of equal total weight are considered, so that

$$
\int_{0}^{l} m(x) d x=M_{0} \text {. }
$$

The governing equation is assumed to have the form

$$
\left[s(x) y^{\prime \prime}(x)\right]^{\prime \prime}+P y^{\prime \prime}(x)+\lambda_{0} y^{\prime}(x)-\omega^{2} m(x) y(x)-q(x)=0
$$

where $y(x)$ is a deflection or deflection amplitude and a prime denotes differentiation with respect to $x$. The ends of the beam are either built-in $\left(y=y^{\prime}=0\right)$, simply-supported $\left(y=s y^{\prime \prime}=0\right)$, or free $\left(s y^{\prime \prime}=\left(s y^{\prime \prime}\right)^{\prime}=0\right)$ in the absence of axial loading, while the transition conditions are continuity of displacement $y$, slope $y^{\prime}$, bending moment $s y^{\prime \prime}$, and shear force $\left(s y^{\prime \prime}\right)^{\prime}$.

In order to obtain an approximate expression for the frequency, displacement or critical load, consider the functional $V$ defined by

$$
\begin{aligned}
V=\int_{0}^{l}\left\{s\left(y^{\prime \prime}\right)^{2}-P\left(y^{\prime}\right)^{2}+2 \lambda_{0} y^{\prime} y-\omega^{2} m y^{2}-\right. & 2 q y\} d x \\
& +\left[y\left(s y^{\prime \prime}\right)^{\prime}-y^{\prime} s y^{\prime \prime}+P y^{\prime} y\right]_{x=0}^{x-l} .
\end{aligned}
$$

For some problems $V$ is stationary with respect to kinematically admissible changes in $y$, while in others this property holds if a constraint is added in the variation of $V$. In either case, a Ritz type of procedure is applicable. The displacement $y(x)$ is assumed

* Received September 11, 1971. This research was supported in part by the U.S. Army Research Office-Durham and in part by the United States Navy under Grant No. NONR N00014-67-A-0191-0009. 
to have the form

$$
y(x)=\sum_{i=1}^{n} a_{i} f_{i}(x)
$$

where each $f_{i}$ satisfies the kinematic boundary and transition conditions. In addition, the stiffness $s(x)$ is restricted to have a certain form characterized by a set of parameters $b_{i}, j=1,2, \cdots, m$. From the equations

$$
\partial V / \partial a_{i}=0, \quad i=1,2, \cdots, n,
$$

along with any required constraint on the variation, one can obtain an approximate expression for the objective function in terms of the $b_{i}$. This expression is then minimized or maximized subject to (2), and the resulting values of $b_{i}$ lead to an approximation for the optimal distribution of stiffness and mass.

This procedure will be demonstrated with some simple examples in which the mass and stiffness are assumed to be either segmentwise constant or segmentwise linear.

Consider first a beam which is to be designed such that the maximum deflection caused by a distributed load $q(x)$ is to be minimized. In this case

$$
V=\int_{0}^{l}\left\{s\left(y^{\prime \prime}\right)^{2}-2 q y\right\} d x .
$$

With the use of (5) and (6), one obtains the linear equations

$$
\sum_{k=1}^{n}\left[\int_{0}^{l} s\left(b_{j}\right) f_{i}^{\prime \prime} f_{k}^{\prime \prime} d x\right] a_{k}=\int_{0}^{l} q f_{i} d x, \quad i=1,2, \cdots, n
$$

which can be solved for the $a_{i}$ in terms of the $b_{i}$. Eq. (5) then furnishes an approximate deflection $y\left(x ; b_{i}\right)$ and the condition $d y / d x=0$ locates the point $x_{0}\left(b_{i}\right)$ of maximum displacement (unless it occurs at a free end). One can eliminate $b_{1}$ with the use of (2), and the remaining $b_{i}$ are then determined from the equations

$$
\left(d / d b_{i}\right)\left|y\left(x_{0} ; b_{i}\right)\right|=0, \quad j=2,3, \cdots, m
$$

so as to minimize the maximum displacement.

For example, consider a beam that is built-in at $x=0$, simply-supported at $x=l$, and subjected to a uniformly distributed load $q(x)=q_{0}$. The stiffness is assumed to be of the form

$$
\begin{aligned}
s(x)=b_{1} & \text { for } \quad 0 \leq x<l / 2 \\
& =b_{2} \text { for } \quad l / 2<x \leq l
\end{aligned}
$$

and the deflection is chosen as a cubic polynomial in each half of the span with the kinematic conditions and moment continuity condition satisfied. The procedure outlined above leads to an optimum ratio $b_{1} / b_{2}=0.92$ for the stiffness parameters.

One can treat beams subjected to loads which vary harmonically in time in a similar manner. If the load is given by $q(x) \cos \omega t$ and the steady-state deflection by $y(x) \cos \omega t$, the functional $V$ becomes

$$
V=\int_{0}^{l}\left\{s\left(y^{\prime \prime}\right)^{2}-\omega^{2} m y^{2}-2 q y\right\} d x .
$$


For instance, consider a cantilever beam under a uniformly distributed load $q_{0} \cos \omega t$ and let the stiffness have the form (10) where $x=0$ is the built-in end. The deflection is taken as a fifth-order polynomial in each half of the beam with all boundary and transition conditions satisfied except for zero shear force at the free end. Eqs. (2), (6) and (9) with $x_{0}=l$ lead to the optimum design for minimum tip deflection amplitude $y(l)$. Define the quantities

$$
\epsilon=\omega^{2} b^{2} l^{4}, \quad \delta=0.5 a^{2} l /\left(M_{0}-a^{2} l\right)
$$

where $a^{2}$ and $b^{2}$ are defined by (1). For $\delta=0.1$ and several values of $\epsilon$, the optimum ratios $b_{1} / b_{2}$ are shown in Table 1 . Also shown are the ratios of the minimum $y(l)$ to that for a uniform beam $\left(b_{1}=b_{2}\right)$ having the same total weight. The tip deflection can be substantially reduced by a simple nonuniform design, especially when the loading frequency is close to a natural frequency of the uniform beam.

A related problem is that of maximizing the fundamental natural frequency of a beam. The appropriate functional is given by (11) with $q=0$. In this case, Eqs. (6) are homogeneous in the $a_{i}$. Setting the determinant of the coefficients of $a_{i}$ equal to zero yields the characteristic equation and the fundamental frequency $\omega_{1}\left(b_{i}\right)$. Again, $b_{1}$ can be eliminated using (2) and $\omega_{1}$ can then be maximized with respect to the remaining $b_{j}$.

It is often desirable to design a structure for maximum stability. If a conservative axial load acts on the beam-column, the functional is given by

$$
V=\int_{0}^{l}\left\{s\left(y^{\prime \prime}\right)^{2}-P\left(y^{\prime}\right)^{2}\right\} d x .
$$

The buckling load $P$ can be maximized in a manner similar to that used above with the fundamental frequency. For example, if the beam-column is built-in at $x=0$ and simplysupported at $x=l$, with $s(x)$ given by (10) and cubic polynomials assumed for the deflection, one arrives at the optimum ratio $b_{1} / b_{2}=0.82$ for the stiffness parameters.

For a system subjected to nonconservative loading, the critical value of the loading parameter for instability often must be determined by a dynamic analysis [17]. As an example, consider a panel flutter problem in which the critical value $\lambda^{*}$ of the aerodynamic parameter $\lambda$ is to be maximized. The governing equation is assumed to be [13]

$$
\left(s y^{\prime \prime}\right)^{\prime \prime}+\lambda_{0} y^{\prime}-\omega^{2} m y=0
$$

where all quantities are now in nondimensional terms, and the edges $x=0$ and $x=l$ are simply-supported. The quantities $m(x)$ and $s(x)$ are assumed to be proportional to the thickness $t(x)$ of the face sheets and, in the direction $x$ of the airflow, are assumed to vary linearly over each of four segments of length $l / 4$ and to be continuous and symmetric about $x=l / 2$. In other words, the thickness $t(x)$ can be characterized by the parameters $b_{0}, b_{1}$ and $b_{2}$ where $t(0)=t(l)=b_{0}, t(l / 4)=t(3 l / 4)=b_{1}$ and $t(l / 2)=b_{2}$.

\section{TABLE 1}

Designs for minimum dynamic deflection

\begin{tabular}{crrrrrr}
\hline$\epsilon$ & 0.0 & 0.1 & 0.2 & 0.3 & 0.4 & 0.5 \\
$b_{1} / b_{2}$ & 3.87 & 4.25 & 4.62 & 5.00 & 5.38 & 5.76 \\
deflection ratio & 0.74 & 0.67 & 0.58 & 0.48 & 0.36 & 0.23 \\
\hline
\end{tabular}


In order to avoid a design with zero thickness, let $b_{0}$ be specified by $b_{0}=0.5 b_{\mathbf{w}}$ where $b_{\mathbf{w}}$ is the thickness of the corresponding uniform face sheet with the same total weight.

The appropriate functional for this case is

$$
V=\int_{0}^{l}\left\{s\left(y^{\prime \prime 2}+2 \lambda_{0} y^{\prime} y-\omega^{2} m y^{2}\right\} d x\right.
$$

and in the application of Eq. (6) the quantity $y^{\prime}$ is treated as a constant (see [18]). The deflection amplitude is taken as

$$
y(x)=a_{1} \sin (\pi x / l)+a_{2} \sin (2 \pi x / l)
$$

which satisfies all kinematic and static conditions except continuity of shear force at $x=l / 4, l / 2$ and $3 l / 4$. Eq. (6) yields homogeneous equations in $a_{1}$ and $a_{2}$, from which one obtains the characteristic equation for $\omega^{2}$. The condition for a double root in $\omega^{2}$ furnishes the critical value $\lambda^{*}\left(b_{i}\right)$, and this can then be maximized subject to (2). The resulting optimal values of the thickness are $b_{1}=2.5 b_{0}$ and $b_{2}=2.0 b_{0}$, so that the optimal design has a "dip" in the center. The increase in $\lambda^{*}$ over that of a uniform panel is 10.1 percent.

Weisshaar [13] has considered the inverse formulation of this panel flutter problem, minimizing the total weight while keeping $\lambda^{*}$ constant. In that form, the procedure above leads to values of $b_{1}$ and $b_{2}$ within 0.5 percent of those found by Weisshaar, even though the approximate $\lambda^{*}$ derived here is in error by about 20 percent. A similar trend has been found in other examples. That is, the procedure used in this note may lead to a good approximation of the optimal design even though the accuracy of the pertinent displacement, frequency or critical load obtained during the calculations may be rather poor. It is not the accuracy of the approximate objective function which is important here, but rather the manner in which this function changes with variations in the design.

In conclusion, then, a Ritz type of method combined with a parametric form of the mass distribution may furnish a useful first approximation to the solution of some static and dynamic optimal structural design problems.

\section{REFERENCES}

[1] C. Y. Sheu and W. Prager, Minimum-weight design with piecewise constant specific stiffness, J. Optimization Theory and Applications 2, 179-186 (1968)

[2] R. T. Shield and W. Prager, Optimal structural design for given deflection, Zeit. angewandte Math. Phys. 21, 513-523 (1970)

[3] L. J. Icerman, Optimal structural design for given dynamic deflection, Int. J. Solids Struct. 5, 473-490 (1969)

[4] Z. Mróz, Optimal design of elastic structures subjected to dynamic, harmonically-varying loads, Zeit. angewandte Math. Mech. 50, 303-309 (1970)

[5] R. H. Plaut, Optimal structural design for given deflection under periodic loading, Quart. Appl. Math. 29, 315-318 (1971)

[6] R. M. Brach, Minimum dynamic response for a class of simply supported beam shapes, Int. J. Mech. Sci. 10, 429-439 (1968)

[7] R. H. Plaut, On minimizing the response of structures to dynamic loading, Zeit. angewandte Math. Phys. 21, 1004-1010 (1970)

[8] F. I. Niordson, On the optimum design of a vibrating beam, Quart. Appl. Math. 23, 47-53 (1965)

[9] M. J. Turner, Design of minimum mass structures with specified natural frequencies, AIAA J. 5, 406$412(1967)$

[10] W. Prager and J. E. Taylor, Problems of optimal structural design, J. Appl. Mech. 35, 102-106, (1968) 
[11] R. M. Brach, On the extremal fundamental frequencies of vibrating beams, Int. J. Solids Struct. 4, 667-674 (1968)

[12] C. Y. Sheu, Elastic minimum-weight design for specified fundamental frequency, Int. J. Solids Struct. 4, 953-958 (1968)

[13] T. A. Weisshaar, An application of control theory methods to the optimization of structures having dynamic or aeroelastic constraints, SUDAAR No. 412, Oct. 1970, Department of Aeronautics and Astronautics, Stanford University

[14] J. B. Keller, The shape of the strongest column, Arch. Rat. Mech. Anal. 5, 275-285 (1960)

[15] I. Tadjbakhsh and J. B. Keller, Strongest columns and isoperimetric inequalities, J. Appl. Mech. 29, 159-164 (1962)

[16] J. E. Taylor, The strongest column: an energy approach, J. Appl. Mech. 34, 486 (1967)

[17] M. Zyczkowski and A. Gajewski, Optimal structural design in non-conservative problems of elastic stability, in Proceedings of the IUTAM Symposium on Instability of Continuous Systems (H. Leipholz, ed.), Springer-Verlag, Berlin, 1971, pp. 295-301

[18] M. Levinson, Application of the Galerkin and Ritz methods to nonconservative problems of elastic stability, Zeit. angewandte Math. Phys. 17, 431-442 (1966) 